\title{
«Von Äpfeln und Birnen»- oder: Warum Krankengeschichte nicht gleich Krankengeschichte ist
}

\author{
Erklärung der FMPP (Verbindung psychiatrisch-psychotherapeutisch tätiger Ärztinnen und Ärzte, \\ Dachverband der SGPP und der SGKJPP) zum PIK-Entscheid Nr. 04043 bezüglich Positionen 00.0140 \\ und 02.0070 «Leistung in Abwesenheit des Patienten»
}

Dr. med. Giampiero Enderli ${ }^{a}$, Dr. med. Patrick Haemmerle ${ }^{b}$, Dr. med. Hans Kurt ${ }^{c}$

Die Psychiater* rechnen nur mit Zeitpositionen ab. Deshalb hat die Position «Leistung in Abwesenheit des Patienten» (02.0070) für diese Fachgruppe eine existentielle Bedeutung. Im folgenden Argumentarium will die FMPP den Unterschied zwischen der psychiatrisch-psychotherapeutischen Position «Leistung in Abwesenheit des Patienten» (02.0070) und der analogen Grundposition (00.0160) darlegen. Den Psychiater-Psychotherapeuten möge dieser Beitrag bei eventuellen Nachfragen von Krankenkassen als Erklärungshilfe dienen.

Das Argumentarium stellt den Entscheid der Paritätischen Interpretationskommission (PIK), wonach das Studium und das Führen der Krankengeschichte Teil der Konsultation seien, nicht in Frage. Allerdings ist santésuisse mit einer unilateralen, nicht abgestimmten und in der Meinung der FMPP unzutreffenden Interpretation dieses PIK-Entscheides an die Öffentlichkeit getreten, weshalb die FMPP mit der unten abgedruckten «Kurzmitteilung» die Kolleginnen und Kollegen auffordert, sich weiterhin an die in den RE-2-Verhandlungen explizit akzeptierte Auslegung der Position 02.0070 zu halten und sie entsprechend abzurechnen.

\footnotetext{
a Präsident Ständige Tarifkommission FMPP

b Präsident FMPP und SGKJPP

c Präsident SGPP

1 TARMED Suisse. Entscheid der Paritätischen Interpretationskommission. Schweiz Ärztezeitung 2004;85(49):2625.

* Die männlichen Bezeichnungen beziehen sich immer auch auf Frauen.

** 00.0010-80 für die Grundversorger, 02.0010-60 für die PsychiaterPsychotherapeuten.
}

Korrespondenz:

FMPP-Sekretariat

Postgasse 17

CH-3011 Bern

Tel. 0313138833

Fax 0313138899

E-Mail: fmpp@psychiatrie.ch

Internet: www.psychiatrie.ch chen Regeln. Für sie alle ist so das Studium und das Führen der Krankengeschichte tarifmässiger Bestandteil der von ihnen durchgeführten Konsultationen. Die Krankengeschichte ist ein gesetzlich vorgeschriebenes Dokument des Arztes, in welches der Patient auch Einsichtsrecht hat. Es beinhaltet z. B. die vom Patienten geschilderten Symptome, die klinischen Befunde, die Anordnung und die Ergebnisse von Untersuchungen, die diagnostischen Überlegungen sowie die Verordnung und die Erfolgskontrolle der Therapie.

Die Positionen der die spezialärztliche Tätigkeit abbildenden 37 Kapitel des TARMED vervollständigen die erwähnten, für alle Ärzte gültigen tarifarischen Regeln. Charakteristisch für das Kapitel 02 der Kinder- und Erwachsenenpsychiater/-psychotherapeuten ist, dass deren spezialärztliche Tätigkeit in einem reinen Zeittarif erfasst wird. Eine Verschiebung der Positionsanteile der verrechenbaren Gesamtarbeitszeit bringt den Leistungsträgern keinerlei Ersparnis. Diese Tatsache gibt dem bemühenden Seilziehen um einen kleineren oder grösseren Anteil der Position 02.0070 an der erbrachten Leistung in Psychiatrie/Psychotherapie einen absurden Anstrich.

Die folgenden Ausführungen zur psychiatrisch-psychotherapeutischen Tätigkeit - die, neben der Arbeit mit dem Patienten selbst, auch die Vor- und Nachbereitung der Behandlung umfasst - sollen die Bedeutung der Position 02.0070 für unsere Arbeitsweise deutlich machen:

Als erste verdient die Rolle und der Stellenwert der Psychotherapie in unserer Disziplin Erwähnung. Der Doppeltitel «Psychiatrie-Psychotherapie» hat in unserem Land eine lange Tradition und hat sich als sinnvoll und hilfreich erwiesen, wobei natürlich jeder Spezialist die Anteile der beiden Komponenten anders gewichtet. Eine Trennung dieser beiden Elemente wird 
dank wissenschaftlicher Erkenntnisse zunehmend obsolet, sind doch auf der einen Seite heutzutage pharmakologische Behandlungen von Leiden möglich, die früher als Domäne der Psychotherapie galten (wie etwa den Angst-, den Zwangskrankheiten und den Depressionen), während auf der anderen Seite psychotherapeutische Methoden auch im Bereich von eher biologienahen, «endogenen» bzw. organischen Leiden immer wichtiger werden, wie z.B. bei Schizophrenien und Demenzkrankheiten.
Zur Psychotherapie hinzu kommt zunehmend vordringlich und oft obligat der Einbezug der sozialen Umwelt, d.h. der Bezugspersonen im familiären Nahraum, im Schul-, Arbeits- und Freizeitbereich, sowie weiterer Helfer und Behandler im sozialpsychiatrischen Netz.

Wohl mehr als in den meisten anderen Fachgebieten finden deshalb in der psychiatrischpsychotherapeutischen Arbeit Interventionen auf diesen drei Achsen statt - der biologischpharmakologischen, der psychologischen und

\section{Position 02.0070 weiter anwenden trotz der verzerrenden Geschichtsschreibung der Versicherungen!}

Kurzmitteilung der STK FMPP

Liebe Kolleginnen, liebe Kollegen

Die Vorkämpfer für einen gerechteren Tarif für die Psychiatrie-Psychotherapie konnten ums Jahr 2000 einen für unser Fach wichtigen Erfolg buchen, als im TARMED-Kapitel 02 eine psychiatriespezifische Position «Leistung in Abwesenheit des Patienten» (LAP) eingeführt wurde. Im Laufe der Reengineeringverhandlungen RE 1 drängten später die Versicherer auf Limitierung dieser Position bei Handabrechnung, wobei sie jedoch ausdrücklich die Berechtigung der Verrechnung der Vor- und Nachbearbeitung psychiatrisch-psychotherapeutischer Behandlungen anerkannten: Die Limitation diente ausschliesslich dazu, die «computerresistenten» Psychiater zur elektronischen Abrechnung zu zwingen. Noch später wurde die von uns in den RE-2-Verhandlungen verlangte weitere Produktivitätssenkung mit der Begründung abgelehnt, dank der Position 02.0070 könnten die Psychiater-Psychotherapeuten ihre Zeit ohne Patienten abrechnen, zu diesem Zwecke sei sie nämlich geschaffen worden. In diesem Sinne wurdet Ihr im folgenden mittels TARMED-Fichen sowie in zahlreichen Mailkontakten immer wieder redundant informiert.

Im vergangenen Oktober liess uns jedoch der PIK-Entscheid Nr. 04043 ein erstes Mal aufhorchen (PIK = Paritätische Interpretationskommission), doch konnte die FMPP in ihrer Stellungnahme erläutern, warum «Äpfel nicht Birnen sind», d.h., warum sich die in ein und demselben PIK-Entscheid zusammen mit der LAP der Grundversorger abgehandelte LAP der Psychiater nicht auf die Vor- und Nachbereitung der psycho- und soziotherapeutischen Behandlungen bezieht.

Doch als wäre santésuisse die PIK in Person, erhalten nun die ersten Kolleginnen und Kollegen massregelnde Briefe mit einer (in einem internen Papier unter «tm-Fragen und Antworten», www.santesuisse.ch/de/srv tarmed_faq.html, nachzulesenden) Auslegung der Position 02.0070, die den wahren Verhandlungsergebnissen eklatant widerspricht. Die santésuisse befindet nämlich, «[...] die Vor-, Auf- und Nachbereitungszeiten eines Psychotherapeuten sind in den Tarifpositionen 02.0010ff und 02.0110ff enthalten und abgegolten. [...] Die [...] tiefere Produktivität [der Psychiater, A.d.A.] lässt sich nur damit begründen, dass diese Vor-, Auf- und Nachbereitungszeiten in den Psychiatriepositionen eingerechnet wurden. Aus der Sicht ist am PIKEntscheid Nr. 04043 festzuhalten.»

Diese geschichtsverzerrende, unilaterale Tarifauslegung der Versicherer darf nie akzeptiert werden, weswegen wir Euch, liebe Kollegin, lieber Kollege, dringend bitten, Eure Abrechnungen in gewohnter Art, d.h. inklusive wahrheitsgetreuen Nachweises der Arbeit ohne Patienten mittels der Position 02.0070, durchzuführen und uns eventuelle Reaktionen der Versicherungen umgehend zu melden.

Mit derselben Post werden wir die FMH um die rückhaltlose Unterstützung unseres Anliegens bitten im Sinne: Wehret den Anfängen!

Mit freundlichen Grüssen
Dr. Patrick Haemmerle Präsident FMPP und SGKJPP
Dr. Hans Kurt Präsident SGPP
Giampiero Enderli Präsident STK 
der sozialen - und müssen auf spezifische Weise vor- und nachbearbeitet sowie dokumentiert werden. Der zeitgenössische Psychiater-Psychotherapeut (und nicht nur der klassische Psychoanalytiker) hält in z.T. umfangreichen persönlichen Notizen - in welche der Patient nicht wie bei einer klassischen Krankengeschichte Einsichtsrecht hat - die durchgeführten und die geplanten Therapieschritte fest. Auch die durch die Therapie ausgelösten eigenen Gefühle («Gegenübertragung», «Resonanz») gilt es zu beachten und in den Behandlungsplan einzuarbeiten. Weitere Beispiele solcher besonders zeitaufwendiger Vor- und Nachbereitungsleistungen in Abwesenheit des Patienten sind die sozialpsychiatrischen, die kinderpsychiatrischen und verhaltenstherapeutischen Therapiepläne und -protokolle sowie die Vorbereitung und Auswertung systemtherapeutischer Videoaufnahmen, welche ebenfalls zum Instrumentarium vieler Psychiater-Psychotherapeuten gehören. Dieser unerlässliche Aufwand und die dabei entstehende Dokumentation überschreitet deutlich den Umfang einer üblichen Krankengeschichte und wird deshalb durch den PIK-Entscheid nicht beeinflusst.
Ein abschliessender Gedanke zum Zustandekommen von Tarifanpassungen: Jeder tarifwirksame Beschluss einer paritätischen Kommission (PIK, PTK) sollte unseres Erachtens den Tarif verbessern, z. B. durch die Möglichkeit, die ärztliche Arbeit noch wirklichkeitsgetreuer abzubilden. Dies ist auch der Grund, dass die psychiatrischen Fachgesellschaften ihren Kollegen empfehlen, ihre Arbeit transparent darzustellen, d.h. auch die Position 02.0070 korrekt und der effektiven Leistung entsprechend abzurechnen. Es bedeutete einen unannehmbaren Rückschritt für das Tarifwerk, wenn Tarifentscheide die Ärzte einschüchtern und zu transparenzschädigender Verzerrung ihrer Abrechnung zwingen würden, statt den Tarif ihrer Arbeitsrealität anzupassen.

Nur die in enger Zusammenarbeit mit den betroffenen Fachgesellschaften durchgeführte, kontinuierliche Weiterentwicklung eines transparenten und die ärztliche Tätigkeit möglichst differenziert und exakt abbildenden und so auch verhandelbaren und anpassbaren Tarifwerks kann zu grösserer Kostengerechtigkeit und damit auch zu Ersparnissen führen. 